\title{
Future of Open Source systems
}

\author{
Karel Charvát \\ Czech Centrum for Science and Society \\ charvat@ccss.cz
}

Keywords: Open Source, Licensing, FOSS base business models. SWOT analysis, Knowledge society, Knowledge economy

\begin{abstract}
Software distribution strategies have many aspects and can be analysed by reviewing different incisions of a strategy. The focus of this paper is on Licensing aspect involves licensing strategy, licensing risks, licensing enforcement costs. Furthermore, by formulating licensing strategy main technical and logistical aspects are predicted also. The key issues of this paper are different business modes for FOSS software and also SWOT analysis of usage and development of FOSS software from point of view of different user groups. This analysis was provided as part of work of Humboldt IP and collaborative@rural IP. Currently this strategy are important issue of members of Czech Centre for Science and Society and WirelessInfo Living Lab, where the models based on dual licensing are key strategy.
\end{abstract}

\section{Introduction}

On one side Open Source software start to play more and more important role on market of geospatial solution, on opposite side, till now you can hear very often argument, that Open Source software is not suitable for large projects. This argument is very often used by producers of closed commercial solution, but also very often public tenders prefer closed commercial solution. If we will look on situation on the market, we can see two important aspects:

- On one side relatively good market success of Web based solution.

- Almost zero acceptance of Open Source desktop GIS solution by standards users (non FOSS community, mainly formed by people from academic communities).

For the future grow of Open Source, which could be connected only with market grow, it is necessary to look on such models, which will attract producers of basic component to publish their systems as Open Source, but which will give them chance also to have profit from large scale usage of their components. The goal is in principle to build communities, where will be chance also generate profit for primary producers of components. The way is probably dual 
license based on combination of GPL license with proprietary license. From models, which are currently used, the most suitable seems to be model of GeoServer.

\section{Free software or new business model}

Is it Open Source the way of free sharing of software or is it new future business model? I am sure, that on this question doesn't exist one answer, but that there are two groups of people and every group will protect their own ideas. The initial idea coming from Richard Matthew Stallman was idea of free software. The current development on software market demonstrate, that this idea could be stolen by commercial sector and could lead to new situation on market and that Open Source and new Open Source business models. The question is, if there will be chance to find common language between business people on one side and protectors of ideas of Free Software on opposite side. This paper mainly explains position of business sector, the interest of companies developing Open Source and also potential new methods of collaboration. The Free Software ideas protector mainly use such terms like freedom and creativity, for business is main goal end user satisfaction. From point of view of major end user community, the main question is not, is software is Open or not, but if it is good or not. Usually more important question is, if software is interoperable, then if it is Open. On opposite side, there is necessary mentioned, that there are many good ideas and software coming from Open Source community mainly academic community, which could have future strong utilisation on the market, if there will be good cooperation between both groups. In next text, I will be focused mainly on Web based technologies, because my personal opinion, that there is future for Open Source business.

\section{Knowledge society and knowledge economy}

Knowledge society and knowledge economy are two terms, which are very often used now and which are also very often mix together. There are strong differences and these differences are connected with both Open Source approaches. Knowledge societies have the characteristic that knowledge forms a major component of any human activity. Economic, social, cultural, and all other human activities become dependent on a huge volume of knowledge and information. The knowledge economy is a term that refers either to an economy of knowledge focused on the production and management of knowledge or to a knowledge-based economy. The important question is, if it is possible to connect both ideas, knowledge society and knowledge economy. Is the knowledge creative force or good? Knowledge is important for future development of society, but knowledge also could bring advantage on the market. There exist many ideas, that free knowledge could solve any social problems, that it could destroyed social barriers. That free knowledge could help to grow the business. But it is not fully true. Free knowledge is advantages for stronger players on the market.

\section{Living Lab model}

When targeting full impact towards knowledge society and economy it is essential to have an integrated view on the technology development, usability and deployment of new solutions. Living Labs (LLs) - are created to develop the best practices, and the best practices then 
are widely deployed throughout Europe with support of funds from regional, national and European sources. LL is not to be understood as technology integration or test bed. The concept is much wider than that. LLs are referred as innovation, validation and deployment environments. LLs are environments which meld together the technological innovation, the application innovation and the societal experiment of knowledge society and economy. LLs are open environments, where the innovation meets the needs of "real" people, in "real environments" leading to systemic solutions which fully take into account the usability perspective. These environments are large enough enabling the development of sustainable business and societal models, widely deployable outside. LL is more about creating together a process and a suitable environment for the process rather than traditional test environments. It should be a driver towards continuous innovation. To establish these environments, the public sector has a role to initiate the drive, by providing the kernel for further exploitable infrastructure and services. This should be done in conjunction with main industrial players, by sharing the risk and allowing for these societal experiments. Verification and deployment of the spearheads can be carried forward with the support of regional, structural and local funds, while the private sector would take the responsibility of the product and service development, and the wide deployment of the solutions developed in LLs.

\section{Guarantee Open Source Software Development model sustainable develop- ment of Living Lab}

Current paradigm is that not only development of software, but all knowledge inside of Living Lab has to be built on principle of Open Source. This could of cause speed up innovation inside of Living Lab, but on opposite side, these models doesn't guarantee long time sustainability of Living Lab and cannot be implemented as single unique model. We compare our practical experiences with theoretical results of Humboldt project and we could conclude our experiences into next topics:

There exist real of SME IT developers to use Open Source for building application. As main advantage was mentioned:

- There can be found the program which suits the end user's needs absolutely.

- The end user can be engaged into the development directly and "leave there his own footprint".

- Sometimes the program could be very simple and the end user can easily grasp how it works.

- User can just cut off the usable part of the code and starts his own project on this.

- It is possible to use a source code from another project if both licenses allow that.

On opposite side, there is small interest of SME developers to publish their components as Open Source. As main threats are mentioned:

- According to the open philosophy it is hard to get some fees for the program usage.

- It is necessary to change the business model. Source of money revenue is not the sales of program, but additional services. 
- The user are sometimes quite ungrateful or even rude, so it is hard to deal with them.

- The group can split apart with all the source codes and found the new company, so called „fork". This is mainly caused by personal arguments inside of a team. Or simply rival company can take over the development and introduce better business plan.

- It can happen, that very important developer can leave the company and the right substitution will not be found. The reason for this (leaving the company) may be also very ridicules.

\section{End user point of view}

The end users see as advantage of Open Source, that it can be deployed for free (no fees, no maintenance cost) without restrictions and therefore might be available almost instantly, compared to common procurement procedures and usually open code (source) allows legal changes to fit user's requirements. Also, open source developments usually cater for enduser requirements and develop mainly to address these requirements instead of following some arbitrary requirements by others (such as copy protection features requested by content industry). It is possible easily adapt of the open source framework to the specific needs and programming of additional extensions (modules). Important is aspect, that long term accessibility is usually guaranteed and for an open platform, there are often more open plugin and extensions available. As main disadvantage is mentioned, that support on voluntary basis alone may be insufficient and sometimes the documentation is missing and training of a new staff may be difficult. There are no guarantees and warranties have to be subjects of special consideration, as they are not explicitly regulated.

\section{Recommendation}

The key issue is to be possible offer with Open Source software not only free products, but also to offer commercial services, commercial documentation and training. This is key issue for commercial success of Open Sources. From this point of view is necessary, that primary producer offer also commercial services or there exist members of community, who are able to guarantee this support.

\section{Open Source from point of view of software integrators}

In the analysis provided by Humboldt projects as main advantages of Open Sources were mentioned:

- There is no need to pay for the software nor for support.

- Redistribution of OSS is possible, under many licenses.

- Own products can be distributed with the OSS fully integrated.

- Company can influence the development by providing resources to an open source project or simply address the programmer with specific remarks. 
- There is a chance to take a part in the development process, even if only helping with the translation or so.

- Open source developments are often highly configurable and thus can form a base for commercial developments of many different directions (The Eclipse RCP is used both as a base for GIS and as an IDE, for example).

- Programs are usually done with emphasis on the backwards compatibility, new releases are fully compatible with the older versions.

- There is a social network around the developers and between the end users.

- Under certain circumstances, many libraries or solutions can be integrated into the new product which would not be allowable if the new product was closed source.

- Programs usually use well known standards and don't come with own new standards.

- Source code is being reviewed many times by the community.

As main advantage were mentioned

- For many businesses, high costs incur since their users have difficulties to switch to the open source platform, especially concerning the operating systems and its administrations a result of the previous point, there are still commercial OS prevailing on the workstations. There can be difficulties with communication with the environment especially what's regarding the exchange formats.

- For some customers, the notion of using open source software is unacceptable because of perceived security issuest is practically not possible to get revenue by selling licenses.

There are quite a few different business and usage models based on the various styles of open source licenses. The decision of software integrators about usage of Open Source often depend on concrete license.

\section{GNU GPL}

The positive aspects of GNU GPL license are

- Maintenance of GPL for derivative software thus guaranteeing free access and distribution.

- Most commonly used free software licensing method.

- The integrator can view and use the source code for his development activities.

- The integrator can modify the source code for his products.

- The integrator can provide his software under his own name.

- The integrator will have a good community support.

- Competitors cannot use the software to create closed-source derived products.

As negative aspects it was mentioned

- SDs have to make their modifications available for all who use their products. 
- Libraries wanting to use the SD's product must also be under the GPL, thus possibly limiting acceptance.

\section{GNU LGPL license}

The positive aspects of GNU LGPL license are:

- The integrator can use the software for his products without limitations.

- The integrator can modify the source code for his products without limitations.

- The integrator SD can provide his software under his own name.

- The integrator will have a good community support for the library.

- The integrator can use the product licensed under the LGPL even in proprietary systems.

- The library itself will be updated by the community, since changes have to be contributed back.

As negative aspects it was mentioned

- Permits use of the library in proprietary programs thus enabling the possibility to not access all software that uses the library.

- Competitors might use the software and create concurrence commercial products.

Generally integrators prefer LGLP license against GPL.

\section{$\mathrm{BSD} / \mathrm{MIT}$}

The positive aspects of BDSM/MIT license are:

- SDs can use the source code for their products.

- SDs can modify the source code for their products.

- SDs can provide his software under their own branding.

- SDs can use for open source or closed source products.

- It's possible to gain much revenue with the software products.

- The flexibility towards different business models is unlimited, all business models can be applied.

As negative aspects it was mentioned

- There might be a lesser advancement.

- Licensing can creep away from open source, thus scaring customers who value open source licensing models.

- Generally it could be mentioned that Integrators prefer possibility to use of the library in proprietary programs thus enabling a greater community to use it and also permits 
modifications of the software without the need to republish them. There is danger that many developers will make closed source products, so there might be no good community support.

\section{Conclusion of integrators view}

Software integrators prefer to use other type of license then GPL. This is advantage for them, that they can use these Open Source components inside of their solution, but there is no benefit for Open Source community.

\section{Open Source from point of view of SME tools developers}

In principle there is small interest of SME developers to publish their components as Open Source. As main threats are mentioned:

- According to the open philosophy it is hard to get some fees for the program usage.

- It is necessary to change the business model. Source of money revenue is not the sales of program, but additional services.

- The user are sometimes quite ungrateful or even rude, so it is hard to deal with them.

- The group can split apart with all the source codes and found the new company, so called "fork". This is mainly caused by personal arguments inside of a team. Or simply rival company can take over the development and introduce better business plan.

- It can happen, that very important developer can leave the company and the right substitution will not be found. The reason for this (leaving the company) may be also very ridicules.

Above mentioned points are very important and it is difficult to overcome this opinion. Also our analysis demonstrated that most of useful open source products were in the beginning supported by certain form of public subsidies (direct intervention or development on universities). Our experiences based on eight year of Open Source usage and development from point of view of SMEs could be concluded into next points:

- For successful opening your products as Open Source on the market you need to have certain, strong market position, which guarantee you, that your profit from opening of your solution will be higher, then your potential loses of part of market.

- It could be very useful to open or your older solution or product, which is not main part of your portfolio. This could bring you big marketing profit.

- It is useful to open as an Open Source such product, which could support selling your other products, for example libraries or solutions, which depend on your commercial products.

\section{GNU GPL license}


The advantage of GNU GPL licence for tools developer is possibility to build community of developers. A possibly good community support will make it easier to add new services or to make existing services more efficient. It is guaranteed that licensing will not change and modifications done to the source code for in-house applications do not need to be shared. The main disadvantage is that the efforts invested by the service provider into the software can create advantage also for competitors.

\section{GNU LGPL licence}

The only one advantage of GNU LGPL licence for tools developer is possibility to build community of developers. On other hand, there exist a lot of problems, the main are, that the ermits use of the library in proprietary programs thus enabling the possibility to not access all software that uses the library and that the competitors might use the software and create concurrence commercial products.

\section{BSD/MIT}

Also here is the only one advantage of BDS/MIT licence for tools developer is possibility to build community of developers. The disadvantage are that the licence of fork could be changed on non Open Source, permits use of the library in proprietary programs thus enabling the possibility to not access all software that uses the library, many developers will make closed source products, so there might be no good community support.

\section{Other possibility WirelessInfo licence}

In some cases tools developers prefer open source only imide of certain community for example Living Lab. In many LL exist opinion, that Open Source model cannot be recommended universally. From this reason, we introduced new type of a licence (WirelessInfo licence), which combine both approaches and advantages from commercial development and open source developments. Source code is managed by one organisation as for open source, but it is not generally free. The source is open for other organisation (SMEs) after signature of this licence, which guarantee to initial developer certain amount of money after selling applications, which will used this components. The number of payments is usually limited on selling first 10 or 20 licences, after is usage free. New users cannot distribute source code to third persons.

\section{Conclusion from tools developers' point of view}

From point of view of commercial tools developers only GPL licence could in principle bring some advantages and could support market position of developers. All other licence give more disadvantages then advantages.

Geinformatics FCE CTU 2009 


\section{Conclusion}

The previous analyses show, that Open Source development could bring a lot of advantages not only for research, but also for commercial community. Mainly in this period of economical crisis, using open source could help for both, users (private and public), but also for all IT sector. There is fact, that on one side, the commercial software is currently extremely expensive and for end users it is difficult to pay license fee for commercial software. The European Commission promote to use open source and usually there are the some recommendation on national levels. On other side usually the conditions of tenders are such, that Open Source producers are excluded from tendering process. There is very often used argument of low quality of Open Source products. Nevertheless this period of economical crises brings excellent opportunity to change situation inside of ICT sector. The situation give more opportunity for Open solution and also for smaller flexible companies, which will be able to adopt their behaviour and react on new situation. Other important issue is large cooperation of small companies and not only inside of one country, but internationally. And it is important also find the way, how this SME commercial sector could cooperate with academic sector. This could be main advantage of Open sources. This all is excellent opportunity for Open source solutions, but to be possible really guarantee user satisfaction, it is necessary to guarantee commercial support and also guarantee more flexible possibilities of licensing. As it was demonstrate earlier, the GPL license could protect interests of developers, but very often is not accepted by integrators. And without strong acceptance of Open Sources by integrators it is difficult to guarantee better position of Open sources on market. So it is important to change basic paradigm of Open source community. The current philosophy (usually called virus or militant) is - to use and contribute. This philosophy is mainly supported by GPL license. My personal opinion is, that this philosophy has to be changed and that the future is in the model - to use and contribute or pay. The best solution for this is combination of GPL and commercial license (with commercial support). Money coming into development could increase power of community. This model is now used for example by GeoServer community. Currently we started to use this model inside of Czech Living Lab, which is organised around WirelessInfo and CCSS.

\section{References}

1. Armantas Ostreika / KTC; Michael Printzos / PRO; Guillermo Schwartz / LOGCMG, Thorsten Reitz/FHG-IGD, Karel Charvat/HSRS, Humboldt, A2.4-D1 Software distribution strategies and business models, 2007

2. arel CHARVAT, Petr HORAK, Sarka HORAKOVA Living Lab (LL) Business Models for Local Development, echallenges 2008, Stockholm

3. Business models analysis for Czech Living Lab (CLL) c@r project internal analysisi

4. WirelessInfo licence

http://www.wirelessinfo.cz

Geinformatics FCE CTU 2009 
